Pacific Journal of Mathematic 


\title{
THE IDEMPOTENTS OF A CLASS OF 0-SIMPLE INVERSE SEMIGROUPS
}

\author{
JANET E. MILLS
}

\begin{abstract}
An $\omega$-semigroup is a semigroup whose idempotents form an $\omega$-chain $e_{0}>e_{1}>e_{2}>\cdots$. In this paper we characterize the semilattice of idempotents of a 0 -simple inverse semigroup whose nonzero $\mathscr{D}$-classes form $\omega$-semigroups.
\end{abstract}

A semilattice $E$ is an interlaced union of $\omega$-chains $C_{\alpha}=$ $\left\{e_{\alpha, 0}>e_{\alpha, 1}>\cdots\right\}, \alpha \in A$, if $E=\bigcup_{a \in A} C_{\alpha}$ and if $\alpha, \beta \in A, i \geqq 0$, then there exists a unique $j \geqq 0$ such that

$$
e_{\beta, j}<e_{\alpha, i} \text { but } e_{\beta, j} \nless e_{\alpha, i+1} .
$$

It will be shown that $Y$ is the semilattice of a 0 -simple inverse semigroup whose nonzero $\mathscr{D}$-classes form $\omega$-semigroups if and only if $Y$ is an interlaced union of $\omega$-chains, with zero adjoined. One such 0 -simple inverse semigroup with semilattice $Y$ will be explicitly displayed.

In the semigroups under consideration, every nonzero $\mathscr{D}$-class is an $\omega$-semigroup, that is, a bisimple $\omega$-semigroup. Since bisimple $\omega$ semigroups were described completely by N. R. Reilly, [8], our semigroups are unions of well-known semigroups; it is the manner in which the idempotents of these $\omega$-semigroups relate to each other that is of interest here. This class of semigroups includes several which have already been explored, for example, simple $\omega$-semigroups, [4] and [7], and certain simple inverse semigroups whose idempotents form the ordinal product of a $\omega$-chain and a semilattice with identity, [6]. Bisimple $\omega$-semigroups occur in abundance within most regular semigroups (see [1]), so it is natural to consider, as a first step, those semigroups whose $\mathscr{D}$-classes are all $\omega$-semigroups.

1. Preliminaries. Let $S$ be an inverse semigroup. For an element $a$ of $S, a^{-1}$ denotes the unique element of $S$ for which $a a^{-1} a=$ $a$ and $a^{-1} a a^{-1}=a^{-1}$. For any subset $D$ of $S, E_{D}$ is the set of idempotents of $S$ contained in $D$. Equivalences $\mathscr{D}$ and $\mathscr{J}$ denote the usual Green's relations.

For inverse semigroups, the property of being 0 -simple is easily seen to be equivalent to the condition: if $e$ and $f$ are nonzero idempotents then there exists an idempotent $g$ such that $g \leqq f$ and $g \mathscr{D} e$, where $\leqq$ is the usual partial order on idempotents.

Let $e$ and $f$ be idempotents with $e \mathscr{D} f$. Then there exists $a$ in 
$S$ such that $a a^{-1}=e$ and $a^{-1} a=f$. Furthermore, the mapping $\sigma_{a}: x \rightarrow$ $a^{-1} x a$ is an isomorphism of $E_{S} e$ onto $E_{S} f$, [3].

The following result is crucial to our development of the structure of the semigroups under consideration.

LEMMA 1.1. Let $S$ be an inverse semigroup in which every nonzero $\mathscr{D}$-class is an $\omega$-semigroup. Then $S$ is 0 -simple if and only if for any two distinct nonzero $\mathscr{D}$-classes $D, D^{\prime}$, if $g, h \in E_{D}$ with $g<h$, then there exists $d \in E_{D^{\prime}}$ such that $d<h$ but $d \nless g$.

Proof. Let $S$ be 0 -simple and $D, D^{\prime}$ be two distinct nonzero $\mathscr{D}$-classes with $g<h, g, h$ in $E_{D}$. By 0 -simplicity, there exists $e \in E_{D^{\prime}}$ such that $e<g$. Since $E_{D^{\prime}}$ is inversely well-ordered, $e$ can be picked to be the maximal idempotent of $D^{\prime}$ beneath $g$. Moreover, since there is an idempotent of $D$ below $e$, there are only a finite number above $e$, so we let $g^{\prime}$ be the minimal such one. That is,

$$
e<g^{\prime} \leqq g<h .
$$

Since $g^{\prime} \mathscr{D} h$, there exists $a$ in $S$ with $a a^{-1}=h, a^{-1} a=g^{\prime}$. Now $a^{-1} e a \mathscr{D} e$ and $a^{-1} e a<g^{\prime}<g$. By maximality of $e$, it follows that $a^{-1} e a \leqq e<g^{\prime}$. If $a^{-1} e a=e$, then $\sigma_{a}$, as defined above, acts in the following manner: $\sigma_{a}(h)=g^{\prime}, \sigma_{a}(e)=e$ and $\sigma_{a}(g)=g^{\prime \prime}$ for some $g^{\prime \prime} \mathscr{D} g$. Since $e<g<h$, then $e<g^{\prime \prime}<g^{\prime}$. But by minimality of $g^{\prime}$, this is impossible. Thus $a^{-1} e a<e<g^{\prime}$.

Since $\sigma_{a^{-1}}$ is also an isomorphism, $a^{-1} e a<e<g^{\prime}$ implies

$$
a\left(a^{-1} e a\right) a^{-1}<a e a^{-1}<a g^{\prime} a^{-1} .
$$

That is, $e<a e a^{-1}<h$. Consequently $d=a e a^{-1} i s \mathscr{D}$-related to $e$ and $d$ satisfies the condition that $d<h$. Furthermore, since $e$ is the maximal idempotent of $D^{\prime}$ below $g, d \nless g$.

The converse follows directly from the remark preceding Lemma 1.1.

An ideal $I$ is called prime if $a b \in I$ implies $a \in I$ or $b \in I$.

LEMMA 1.2. If $S$ is a 0-simple inverse semigroup whose nonzero $\mathscr{D}$-classes are $\omega$-semigroups then 0 is a prime ideal, and $S \backslash 0$ is a simple inverse semigroup whose $\mathscr{D}$-classes are $\omega$-semigroups.

Proof. Let $e$ and $f$ be nonzero idempotents of $S$ with $e f=0$. Then $e$ and $f$ must be in distinct $\mathscr{D}$-classes, since each $\mathscr{D}$-class is closed. By 0 -simplicity, there exists an idempotent $g$ such that $g \leqq e$ and $g \mathscr{D} f$. Since $f$ and $g$ are in an $\omega$-semigroup, either $g \leqq f$ or 
$f<g$. But if $f<g$, then $f \leqq e$ and $e f \neq 0$. Hence $g \leqq f$ and $g \leqq e$. But this implies that $g \leqq e f=0$. But $g \neq 0$, and thus $e f \neq 0$. Therefore, 0 is a prime ideal of $E_{S}$, and thus of $S$.

2. The idempotent structure. In light of Lemma 1.2, we now restrict ourselves to simple inverse semigroups whose $\mathscr{D}$-classes are $\omega$-semigroups. In such a semigroup, we now show that the semilattice of idempotents is an interlaced union of $\omega$-chains.

Lemma 2.1. Let $S$ be a simple inverse semigroup whose $\mathscr{D}$ classes are $\omega$-semigroups $D_{\alpha}, \alpha \in A$, and $E_{D_{\alpha}}=\left\{e_{\alpha, 0}>e_{\alpha, 1}>\cdots\right\}$. The following properties hold in $E_{S}$.

(i) If $e_{\alpha, i} \leqq e_{\beta, j}$ then $i \geqq j$.

(ii) For $\alpha, \beta \in A, i, j \geqq 0$, and for all $n$ such that $-j \leqq n<+\infty$,

$$
e_{\alpha, i}<e_{\beta, j} \longleftrightarrow e_{\alpha, i+n}<e_{\beta, j+n} \text {. }
$$

(iii) If $e_{\alpha, i} e_{\beta, j}=e_{\gamma, k}$ then $e_{\alpha, i+n} e_{\beta, j+n}=e_{\gamma, k+n}$, for all $n \geqq-\min \{i, j\}$.

(iv) For $\alpha \in A$, if $a a^{-1}=e_{\alpha, i}, a^{-1} a=e_{\alpha, j}$ then $\sigma_{a}: E e_{\alpha, i} \rightarrow E e_{\alpha, j}$ defined by $x \sigma_{a}=a^{-1} x a$, is an isomorphism such that if $e_{\beta, k} \leqq e_{\alpha, i}$, then

$$
e_{\beta, k} \sigma_{a}=e_{\beta, k+(j-i)} \text {. }
$$

Proof. (i) Let $e_{\alpha, i}<e_{\beta, j}$. Consider the set

$$
M=\left\{k \mid e_{\alpha, k}<e_{\beta, 0}, e_{\alpha, k} \nless e_{\beta, j}\right\} .
$$

Then if $k$ is in $M, k<i$ since $e_{\alpha, i}<e_{\beta, j}$. On the other hand, by Lemma 1.1, for all $p<j$, there exists $p^{\prime}$ such that $e_{\alpha, p^{\prime}}<e_{\beta, p}, e_{\alpha, p^{\prime}} \nless$ $e_{\beta, p+1}$; each $p^{\prime}$ is in $M$ and they are all distinct. Consequently $j-1 \leqq$ $|M|<i$, so $i>j$.

We know from [3] that $\sigma_{a}$ is an isomorphism and thus preserves $\mathscr{D}$-classes. Therefore, if $e_{\beta, k} \leqq e_{\alpha, i}$, then $e_{\beta, k} \sigma_{a}=e_{\beta, m}$ for some $m$. In addition it is clear that for $e_{\alpha, k} \leqq e_{\alpha, i}, e_{\alpha, k} \sigma_{a}=e_{\alpha, k+(j-i)}$, since there must be a one-to-one correspondence between the sets $\left\{e_{\alpha, k}<\cdots<e_{\alpha, i}\right\}$ and $\left\{e_{\alpha, k} \sigma_{a}<\cdots<e_{\alpha, j}\right\}$. The proof of (1) for arbitrary $\beta$ will be made after (ii) and (iii) are proved.

(ii) Let $e_{\alpha, i}<e_{\beta, j}$. It will first be shown that $e_{\alpha, i+1}<e_{\beta, j+1}$. Either $e_{\alpha, i}<e_{\beta, j+1}$ and thus $e_{\alpha, i+1}<e_{\alpha, i}<e_{\beta, j+1}$, or $e_{\alpha, i} \nless e_{\beta, j+1}$. We may assume the latter. By simplicity, there exists $e_{\beta, k}<e_{\alpha, i}$, so let $r=\min \left\{k \mid e_{\beta, k}<e_{\alpha, i}\right\}$. That is, using 1.1

$$
e_{\beta, r}<e_{\alpha, i}<e_{\beta, j} \text { and } e_{\beta, r} \nless e_{\alpha, i+1} \text {. }
$$

Let $a a^{-1}=e_{\beta, j}$ and $a^{-1} a=e_{\beta, j+1}$. Then

$$
a^{-1} e_{\beta, r} a<a^{-1} e_{\alpha, i} a<a^{-1} e_{\beta, j} a,
$$


where the strict inequalities hold since $\sigma_{a}$ is an isomorphism. That is,

$$
e_{\beta, r+1}<a^{-1} e_{\alpha, i} a<e_{\beta, j+1}
$$

since $e_{\beta, r} \sigma_{a}=e_{\beta, r+1}$ as we have seen earlier. Now $a^{-1} e_{\alpha, i} a \mathscr{D} e_{\alpha, i}$ and thus $a^{-1} e_{\alpha, i} a<e_{\alpha, i}$ since $e_{\alpha, i} \nless e_{\beta, j+1}$. If $a^{-1} e_{\alpha, i} a<e_{\alpha, i+1}$ then by 1.1, there exists $p$ such that $e_{\beta, p}<e_{\alpha, i+1}, e_{\beta, p} \nless a^{-1} e_{\alpha, i} a$. By definition of $r, p \geqq r$ and in fact $p>r$ since $e_{\beta, r} \nless e_{\alpha, i+1}$. But then by (2) $e_{\beta, p} \leqq e_{\beta, r+1}<a^{-1} e_{\alpha, i} a$, contrary to the assumption. Hence $a^{-1} e_{\alpha, i} a=$ $e_{\alpha, i+1}$ and thus $e_{\alpha, i+1}<e_{\beta, j+1}$.

That $e_{\alpha, i+n}<e_{\beta, j+n}$ for all $n \geqq 0$ follows by induction.

Now consider the case $n=-1$. Let $j>0$. Then $i>j>0$ by (i). Either $e_{\alpha, i}$ is the maximal idempotent of $D_{\alpha}$ less than $e_{\beta, j}$, or $e_{\alpha, i}<e_{\alpha, i-1}<e_{\beta, j}<e_{\beta, j-1}$. Thus we may assume that the former holds. By 1.1, there exists $m$ such that $e_{\alpha, m}<e_{\beta, j-1}, e_{\alpha, m} \nless e_{\beta, j}$. Since $e_{\alpha, i}<$ $e_{\beta, j}$, it follows that $m \leqq i-1$. Hence $e_{\alpha, i-1} \leqq e_{\alpha, m}<e_{\beta, j-1}$. The proof for $n$ such that $-j \leqq n \leqq-1$ is by induction.

(iii) The proof of (iii) is made using repeated applications of (ii).

To see that (1) holds for arbitrary $\beta$, let $\sigma_{a}$ be defined as in (iv). Then, as we have stated, for $e_{\beta, k}<e_{\alpha, i}, a^{-1} e_{\beta, k} a=e_{\beta, p}$ for some $p$. By (ii), $e_{\beta, k}<e_{\alpha, i}$ if and only if $e_{\beta, k+(j-i)} \leqq e_{\alpha, i+(j-i)}=e_{\alpha, j}$. Since $\sigma_{a}$ is one-to-one and preserves $\mathscr{D}$-classes, $e_{\beta, k} \sigma_{a}=e_{\beta, k+(j-i)}$.

THEOREM 2.2. If $S$ is a simple inverse semigroup whose $\mathscr{D}$ classes are $\omega$-semigroups, then $E_{S}$ is an interlaced union of $\omega$-chains.

Proof. We know that $E_{S}$ is a union of $\omega$-chains $E_{D_{\alpha}}=\left\{e_{\alpha, 0}>\right.$ $\left.e_{\alpha, 1}>\cdots\right\}, \alpha \in A$, where $D_{\alpha}$ is a $\mathscr{D}$-class. Let $\alpha, \beta \in A, i \geqq 0$. We must find a unique $j \geqq 0$ such that $e_{\beta, j}<e_{\alpha, i}, e_{\beta, j} \nless e_{\alpha, i+1}$. Consider the set

$$
K=\left\{j \mid e_{\beta, j}<e_{\alpha, i}\right\} .
$$

By Lemma 1.1, $K$ is nonempty, and thus $K$ must have a least element, call it $m$. Then $e_{\beta, m}<e_{\alpha, i}$. If $e_{\beta, m}<e_{\alpha, i+1}$, then by Lemma 2.1 (ii), $e_{\beta, m-1}<e_{\alpha,(i+1)-1}$. That is, $e_{\beta, m-1}<e_{\alpha, i}$. By minimality of $m$, this is impossible. Thus $e_{\alpha, m} \nless e_{\alpha, i+1}$.

Since $e_{\alpha, i} \mathscr{D} e_{\alpha, i+1}$, there exists $a \in S$ such that $a a^{-1}=e_{\alpha, i}, a^{-1} a=e_{\alpha, i+1}$ and $\sigma_{a}$ defined by $e_{\gamma, k} \sigma_{a}=e_{\gamma, k+1}$ is an isomorphism of $E e_{\alpha, i}$ onto $E_{\alpha, i+1}$, by Lemma 2.1(iv). Now $e_{\beta, m}<e_{\alpha, i}$ so $e_{\beta, m} \sigma_{a}=e_{\beta, m+1}<e_{\alpha, i+1}$. Hence $e_{\beta, k}<e_{\alpha, i+1}$ for all $k>m$. From this and minimality of $m$, it follows that $e_{\beta, m}$ is the unique idempotent in $D_{\beta}$ such that $e_{\beta, m}<e_{\alpha, i}$ and $e_{\beta, m} \nless e_{\alpha, i+1}$. Therefore, $E_{S}$ is an interlaced union of $\omega$-chains $E_{D_{\alpha}}, \alpha \in A$.

3. An interlaced union of $\omega$-chains, Given an interlaced union 
of $\omega$-chains, we now construct a simple inverse semigroup associated with it.

Let $E$ be an interlaced union of $\omega$-chains $e_{\alpha, 0}>e_{\alpha, 1}>\cdots, \alpha \in A$. Recall that this means that for all $\alpha, \beta \in A, i \geqq 0$, there exists a unique $j \geqq 0$ such that $e_{\beta, j}<e_{\alpha, i}, e_{\beta, j} \nless e_{\alpha, i+1}$.

Lemma 3.1. For $E$ as described, the following hold.

(i) If $e_{\alpha, i} \leqq e_{\beta, j}$ then $i \geqq j$.

(ii) If $e_{\alpha, i} \leqq e_{\beta, j}$ then $e_{\alpha, i+n} \leqq e_{\beta, j+n}$ for all $n \geqq-j$.

(iii) If $e_{\alpha, i} e_{\beta, j}=e_{\gamma, k}$ then $e_{\alpha, i+n} e_{\beta, j+n}=e_{\gamma, k+n}$ for all $n \geqq 0$.

Proof. First we prove (ii) for all $n \geqq-\min \{i, j\}$. Assume that $e_{\alpha, i} \leqq e_{\beta, j}$. Let $n \geqq 0$ and assume $e_{\alpha, i+n} \leqq e_{\beta, j+n}$. If $e_{\alpha, i+n} \leqq e_{\beta, j+n+1}$, then $e_{\alpha, i+n+1}<e_{\alpha, i+n} \leqq e_{\beta, j+n+1}$ and the result holds. If $e_{\alpha, i+n} \nless e_{\beta, j+n+1}$ then $e_{\alpha, i+n}$ is the unique element below $e_{\beta, j+n}$ which is not below $e_{\beta, j+n+1}$. Consider $e_{\alpha, i+n+1}$. We know $e_{\alpha, i+n+1}<e_{\beta, j+n}$ since $e_{\alpha, i+n+1}<$ $e_{\alpha, i+n}$; therefore, by uniqueness of $i+n$, we have $e_{\alpha, i+n+1} \leqq e_{\beta, j+n+1}$. By induction, (ii) holds for all $n \geqq 0$.

Now let $n>-\min \{i, j\}$ and let $e_{\alpha, i-n} \leqq e_{\beta, j-n}$. Either $e_{\alpha, i-n-1} \leqq$ $e_{\beta, j-n}<e_{\beta, j-n-1}$, or else $e_{\alpha, i-n-1} \nless e_{\beta, j-n}$. There exists a unique $k \geqq 0$ such that $e_{\alpha, k}<e_{\beta, j-n-1}$ and $e_{\alpha, k} \nless e_{\beta, j-n}$. If $e_{\alpha, i-n-1} \nless e_{\beta, j-n}$ then it must be that $k \leqq i-n-1$ and $e_{\alpha, i-n-1} \leqq e_{\alpha, k}<e_{\beta, j-n-1}$. Consequently, for all $n$ such that $-\min \{i, j\} \leqq n<+\infty$, (ii) holds.

(i) Let $e_{\alpha, i} \leqq e_{\beta, j}$ and assume $i<j$. Then by the above paragraph, $e_{\alpha, i-i} \leqq e_{\beta, j-i}$. That is, $e_{\alpha, 0} \leqq e_{\beta, j-i}<e_{\beta, 0}$. Since $E$ is an interlaced union of $\omega$-chains, there exists $k \geqq 0$ such that $e_{\alpha, k}<e_{\beta, 0}$ and $e_{\alpha, k} \nless e_{\beta, 1}$. But $j-i \geqq 1$ and $e_{\alpha, k} \leqq e_{\beta, 0} \leqq e_{\beta, j-i} \leqq e_{\beta, 1}$. This is impossible. Therefore $i \geqq j$. This also shows that (ii) is true for all $n \geqq-j=-\min \{i, j\}$.

(iii) Let $e_{\alpha, i} e_{\beta, j}=e_{r, k}$. Then $e_{\gamma, k} \leqq e_{\alpha, i}$ and $e_{r, k} \leqq e_{\beta, j}$, so that by (ii), $e_{\gamma, k+1} \leqq e_{\alpha, i+1}, e_{\gamma, k+1} \leqq e_{\beta, j+1}$. That is,

$$
e_{\gamma, k+1} \leqq e_{\alpha, i+1} e_{\beta, j+1}<e_{\alpha, i} e_{\beta, j}=e_{i, k} .
$$

Let $e_{\alpha, i+1} e_{\beta, j+1}=e_{\hat{\delta}, p}$. Then $e_{\hat{\delta}, p} \leqq e_{\alpha, i+1}, e_{\hat{o}, p} \leqq e_{\beta, j+1}$, so by (ii), $e_{\hat{o}, p-1} \leqq$ $e_{\alpha, i}, e_{\hat{\imath}, p-1} \leqq e_{\beta, j}$. That is, $e_{\hat{\delta}, p-1} \leqq e_{\alpha, i} e_{\beta, j}=e_{r, k}$. Consequently, $e_{\gamma, k+1} \leqq$ $e_{\hat{o}, p}<e_{\hat{s}, p-1} \leqq e_{r, k}$. But then by uniqueness in the definition of $E$, both $e_{j, p}$ and $e_{\hat{\delta}, p-1}$ can not be strictly between $e_{r, k+1}$ and $e_{r, k}$. Thus $e_{\hat{\theta}, p}=$ $e_{\gamma, k+1}$ and $e_{\gamma, k+1}=e_{\alpha, i+1} e_{\beta, j+1}$. By induction, (iii) holds for all $n \geqq 0$.

THeOREM 3.2 Let $E$ be an interlaced union of $\omega$-chains $\left\{e_{\alpha, 0}>\right.$ $\left.e_{\alpha, 1}>\cdots\right\}, \alpha \in A$. For $\alpha \in A, m, n \geqq 0$, let $\tau_{(m, \alpha, n)}$ be the mapping from $E e_{\alpha, m}$ onto $E e_{\alpha, n}$ defined by

$$
e_{\beta, j} \tau_{(m, \alpha, n)}=e_{\beta, j+(n-m)}
$$


Then $W=\left\{\tau_{(m, \alpha, n)} \mid \alpha \in A, m, n \geqq 0\right\}$, under composition, is a simple inverse semigroup whose $\mathscr{D}$-classes are $\omega$-semigroups, and $E_{W} \cong E$.

Proof. By Theorem 3.2 of [5], to see that $W$ is a simple inverse semigroup, it suffices to show that $W$ is a subtransitive inverse subsemigroup of $T_{E}$, the set of isomorphisms of principal ideals of $E$. Using (ii) and (iii) of 3.1 , it is not difficult to show that $\tau_{(m, \alpha, n)}$ is an isomorphism of $E e_{\alpha, m}$ onto $E e_{\alpha, n}$, and thus $W$ is contained in $T_{E}$.

To see that $W$ is closed, let $\tau_{(m, \alpha, n)}, \tau_{(i, \beta, j)}$ be in $W$. Certainly $\tau_{(m, \alpha, n)} \tau_{(i, \beta, j)}$ is an isomorphism from one subset of $E$ to another. We need to show its domain is $E e_{\hat{\delta}, p}$ and its range is $E e_{\hat{\delta}, q}$ for some $\delta \in$ $A, p, q \geqq 0$.

Now, $e_{\gamma, k} \in$ domain of $\tau_{(m, \alpha, n)} \tau_{(i, \beta, j)}$ if and only if

$$
e_{\gamma, k} \leqq e_{\alpha, m} \text { and } e_{\gamma, k+(n-m)} \leqq e_{\beta, i},
$$

which by Lemma 3.1 (ii) is equivalent to

$$
e_{\gamma, k} \leqq e_{\alpha, m} \text { and } \quad e_{\gamma, k} \leqq e_{\beta, i-(n-m)} \cdot
$$

This is equivalent to

$$
e_{\gamma, k} \leqq e_{\alpha, m} e_{\beta, i-(n-m)} \text {. }
$$

Thus the domain of $\tau_{(m, \alpha, n)} \tau_{(i, \beta, j)}$ is $E e_{\alpha, m} e_{\beta, i-(n-m)}$.

Now, $e_{\tilde{o}, s}$ is in the range of $\tau_{(m, \alpha, n)} \tau_{(i, \beta, j)}$ if and only if

$$
e_{\tilde{\delta}, s} \leqq e_{\beta, j} \quad \text { and } \quad e_{\tilde{o}, s-(j-i)} \leqq e_{\alpha, n}
$$

which is equivalent to

$$
e_{\hat{\delta}, s} \leqq e_{\beta, j} \quad \text { and } \quad e_{\tilde{\delta}, s} \leqq e_{\alpha, n+(j-i)}
$$

This in turn is equivalent to

$$
e_{\hat{o}, s} \leqq e_{\alpha, n+(i-j)} e_{\beta, j} .
$$

Therefore, the range of $\tau_{(m, \alpha, n)} \tau_{(i, \beta, j)}$ is $E e_{\alpha, n+(j-i)} e_{\beta, j}$.

If $(n-m)+(j-i) \geqq 0$, and $e_{\alpha, m} e_{\beta, i-(n-m)}=e_{\hat{o}, p}$ for some $\delta \in A$, $p \geqq 0$, then by Lemma 3.1 (iii),

$$
e_{\alpha, m+(n-m)+(j-i)} e_{\beta, i-(n-m)+(n-m)+(j-i)}=e_{\delta, p+(n-m)+(j-i)} .
$$

That is,

$$
e_{\alpha, n+(j-i)} e_{\beta, j}=e_{\delta, p+(n-m)+(j-i)}=e_{\hat{o}, q},
$$

for some $q \geqq 0$, and $\tau_{(m, \alpha, n)} \tau_{(i, \beta, j)}=\tau_{(p, \tilde{s}, q)}$. If $(n-m)+(j-i) \leqq 0$, a similar argument works for $e_{\alpha, n+(i-j)} e_{\beta, j}$. Thus $W$ is closed and is a subsemigroup of $T_{E}$. It is clearly an inverse semigroup since $\tau_{(n, \alpha, m)}=\tau_{(m, \alpha, n)}^{-1}$. 
In order that $W$ be subtransitive, it must satisfy the condition: for $e, f$ in $E$, there exists $\theta \in W$ such that domain of $\theta=E e$, range of $\theta \leqq E f$. For $e_{\alpha, i}, e_{\beta, j}$ in $E$, there exists $k \geqq 0$ such that $e_{\alpha, k} \leqq e_{\beta, j}$, since $E$ is interlaced. Thus $\theta=\tau_{(i, \alpha, k)}$ satisfies the necessary condition.

Since idempotents of $W$ are of the form $\tau_{(i, \alpha, i)}, E_{W}$ is an interlaced union of $\omega$-chains, isomorphic to $E$ under the map: $e_{\alpha, i} \rightarrow \tau_{(i, \alpha, i)}$. By Lemma 1.2 of [5], it is clear the $\tau_{(i, \alpha, i)} \mathscr{D} \tau_{(j, \beta, j)}$ if and only if $\alpha=\beta$, so the $\mathscr{D}$-classes of $W$ are $\omega$-semigroups.

THEOREM 3.3. A semilattice $E$ is the semilattice of idempotents of a 0-simple inverse semigroup whose nonzero $\mathscr{D}$-classes are $\omega$ semigroups if and only if $E$ is an interlaced union of $\omega$-chains with 0 adjoined.

Proof. This follows immediately from Corollary 1.2, Theorem 2.2 and Theorem 3.2.

4. An application. The simplest example of an interlaced union of $\omega$-chains is that of an $\omega$-chain itself. The inverse semigroups correspcnding are simple $\omega$-semigroups, the structure of which was determined by Kochin [4] and Munn [7]. The following result demonstrates the strength of the condition imposed on an interlaced union of $\omega$-chains.

THEOREM 4.1. If $S$ is a simple inverse semigroup with exactly two $\mathscr{D}$-classes, each of which is an w-semigroup, then $S$ is itself an $\omega$-semigroup.

Proof. Let $\left\{e_{0}>e_{1}>\cdots\right\}$ and $\left\{f_{0}>f_{1}>\cdots\right\}$ be the idempotents of the two $\mathscr{D}$-classes. Since $E_{S}$ must be an interlaced union of $\omega$ chains by Theorem 2.2 , there exists unique $i \geqq 0, j \geqq 0$ such that

$$
e_{i}<f_{0}, e_{i} \nless f_{1} \text {, and } f_{j}<e_{0}, f_{j} \nless e_{1} \text {. }
$$

Now $e_{0} f_{0} \in E_{S}$ so $e_{0} f_{0}=e_{k}$ or $f_{k}$ for some $k$. Without loss of generality we may assume $e_{0} f_{0}=e_{k}$. Then $e_{k}<f_{0}$. But $e_{i}<f_{0}$ implies that $e_{i}=$ $e_{i} e_{0} \leqq e_{0} f_{0}=e_{k}$, so $i \geqq k$. But if $e_{i}<e_{k}$, then $e_{k} \nless f_{1}$ since $e_{i} \nless f_{1}$. Thus by uniqueness, $k=i$ and $e_{0} f_{0}=e_{i}$. Now $f_{j}<e_{0}$ so $f_{j}<e_{0} f_{0}=e_{i}$. Since $f_{j} \nless e_{1}$, it follows that $i=0$. Hence $e_{0} f_{0}=e_{0}$, i.e., $e_{0} \leqq f_{0}$. By Lemma 3.1(ii), $e_{n} \leqq f_{n}$ for all $n$.

We need to show that $f_{1}<e_{0}$. Since $e_{1}<f_{1}$ and $e_{0}<f_{0}$, then

$$
e_{1} \leqq e_{0} f_{1}<e_{0} f_{0}=e_{0} \text {. }
$$

If $e_{1}=e_{0} f_{1}$ then $f_{j}<e_{0}$ and $f_{j} \nless e_{1}$ implies that $f_{j}=f_{j} f_{0}<e_{0} f_{1}=e_{1}$. But 
this is impossible, so $e_{1}<e_{0} f_{1}<e_{0}$. Thus $e_{0} f_{1}=f_{j}$, by uniqueness, and $e_{1}<f_{j}<e_{0}$. By property (i) of Lemma 3.1, $j \leqq 1$, so $j=1$, and $e_{1}<f_{1}<e_{0}<f_{0}$. By property (ii), this means that $E_{S}$ is an $\omega$-chain.

To see that Theorem 4.1 does not hold for more than two $\mathscr{D}$ classes, consider the following semilattice $E$.

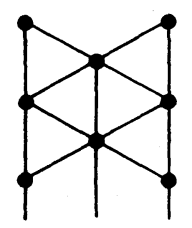

This semilattice $E$ is the interlaced union of three $\omega$-chains, each chain being a column, but $E$ is not an $\omega$-chain itself. For more than three $\mathscr{D}$-classes, one may add to $E \omega$-chains each of whose elements is put between two elements of one of the columns in the semilattice $E$.

\section{REFERENCES}

1. J. E. Ault, Semigroups with bisimple and simple $\omega$-semigroups, Semigroup Forum, 9 (1975), 318-333.

2. A. H. Clifford and G. B. Preston, Algebraic theory of semigroups, Math. Surveys AMS, No. 7, Providence, R. I., Vol. I, 1961.

3. J. M. Howie, The maximal idempotent-separating congruence on an inverse semigroup, Proc. Edinburgh Math. Soc., 14 (1964), 71-79.

4. B. P. Kochin, The structure of inverse ideal-simple $\omega$-semigroups, Vestnik Leningrad Univ., 23 (1968), 41-50 (Russian).

5. W. D. Munn, Fundamental inverse semigroups, Quarterly J. Math. Oxford Series, (2) 21 (1970), 157-170.

6. - On simple inverse semigroups, Semigroup Forum, 1 (1970), 63-74.

7. — Regular w-semigroups, Glasgow Math. J., 9 (1968), 46-66.

8. N. R. Reilly, Bisimple $\omega$-semigroups, Proc. Glasgow Math. Assoc., 7 (1966), 160-167.

Received February 3, 1978.

JAMES MADISON UNIVERSITY

HARRISONBURG, VA 22807 


\section{PACIFIC JOURNAL OF MATHEMATICS}

EDITORS

DONALD BABBITT (Managing Editor)

University of California

Los Angeles, California 90024

HUGo RossI

University of Utah

Salt Lake City, UT 84112

C. C. MOORE

J. DUGUNDJI

Department of Mathematics

University of Southern California

Los Angeles, California 90007

R. FinN AND J. MiLgraM

Stanford University

Stanford, California 94305

University of California

Berkeley, CA 94720

\section{ASSOCIATE EDITORS}

E. F. BrCKENBACH

B. H. NeUmanN

F. WOLF

K. YoshidA

\section{SUPPORTING INSTITUTIONS}

UNIVERSITY OF BRITISH COLUMBIA

UNIVERSITY OF SOUTHERN CALIFORNIA

CALIFORNIA INSTITUTE OF TECHNOLOGY

STANFORD UNIVERSITY

UNIVERSITY OF CALIFORNIA

UNIVERSITY OF HAWAII

MONTANA STATE UNIVERSITY

UNIVERSITY OF TOKYO

UNIVERSITY OF NEVADA, RENO

UNIVERSITY OF UTAH

NEW MEXICO STATE UNIVERSITY

WASHINGTON STATE UNIVERSITY

OREGON STATE UNIVERSITY

UNIVERSITY OF WASHINGTON

UNIVERSITY OF OREGON 


\section{Pacific Journal of Mathematics}

\section{Vol. 81, No. $1 \quad$ November, 1979}

Thomas E. Armstrong, Simplicial subdivision of infinite-dimensional compact cubes ..................................... 1

Herbert Stanley Bear, Jr., Approximate identities and pointwise convergence ................................

Richard David Bourgin, Partial orderings for integral representations on convex sets with the Radon-Nikodým property..................

Alan Day, Herbert S. Gaskill and Werner Poguntke, Distributive lattices

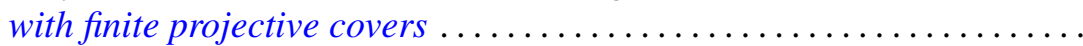

Heneri Amos Murima Dzinotyiweyi and Gerard L. G. Sleijpen, A note on measures on foundation semigroups with weakly compact orbits ......

Ronald James Evans, Resolution of sign ambiguities in Jacobi and Jacobsthal sums ...................................

John Albert Fridy, Tauberian theorems via block dominated matrices ......

Matthew Gould and Helen H. James, Automorphism groups retracting onto

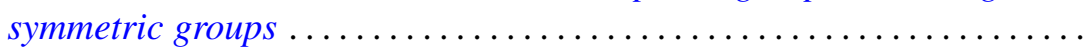

Kurt Kreith, Nonlinear differential equations with monotone solutions . . . . 101

Brian William McEnnis, Shifts on indefinite inner product spaces........ 113

Joseph B. Miles, On entire functions of infinite order with radially

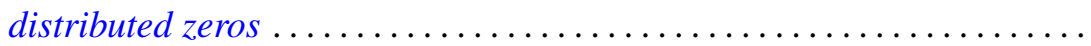

Janet E. Mills, The idempotents of a class of 0-simple inverse semigroups ...............................

Edward Jean Moulis, Jr., Generalizations of the Robertson functions ...

Richard A. Moynihan and Berthold Schweizer, Betweenness relations in probabilistic metric spaces.......................

Stanley Ocken, Perturbing embeddings in codimension two ....

Masilamani Sambandham, On the average number of real zeros of a class of random algebraic curves.

Jerry Searcy and B. Andreas Troesch, A cyclic inequality and a related eigenvalue problem.

Roger R. Smith and Joseph Dinneen Ward, $M$-ideals in $B\left(l_{p}\right)$...

Michel Talagrand, Deux généralisations d'un théorème de I. Namioka ..

Jürgen Voigt, $O n Y$-closed subspaces of $X$, for Banach spaces $X \subset Y$;

existence of alternating elements in subspaces of $C(J)$

Sidney Martin Webster, On mapping an $n$-ball into an $(n+1)$-ball in complex spaces

David J. Winter, Triangulable subalgebras of Lie p-algebras ... 